\title{
Surgical management of posterior petrous meningiomas
}

\author{
James K. Liu, M.D., Oren N. Gottfried, M.D., and William T. Couldwell, M.D., Ph.D. \\ Department of Neurosurgery, University of Utah School of Medicine, Salt Lake City, Utah
}

\begin{abstract}
Posterior petrous meningiomas (commonly termed posterior pyramid meningiomas and/or meningiomas of the posterior surface of the petrous pyramid) are the most common meningiomas of the posterior cranial fossa. They are located along the posterior surface of the temporal bone in the region of the cerebellopontine angle. They often mimic vestibular schwannomas, both clinically and on neuroimaging studies. Common clinical symptoms include hearing loss, cerebellar ataxia, and trigeminal neuropathy. The site of dural origin determines the direction of cranial nerve displacement. Total resection can be achieved in most cases with a low morbidity rate and an excellent prognosis. The authors review the surgical management of posterior petrous meningiomas.
\end{abstract}

\section{KEY WORDS • meningioma • cerebellopontine angle • skull base surgery • petrous bone}

Posterior fossa meningiomas comprise approximately $10 \%$ of all intracranial meningiomas. ${ }^{8}$ Castellano and Ruggiero ${ }^{4}$ reviewed Olivecrona's experience with treating posterior fossa meningiomas and classified them based on the site of dural attachment. They described the location as cerebellar convexity (10\%), tentorium (30\%), posterior surface of the petrous bone (42\%), clivus (11\%), or foramen magnum (4\%). Based on their extensive microsurgical experience, Yaşargil, et al., ${ }^{23}$ proposed a new classification of posterior fossa meningiomas: 1) clival; 2) petroclival; 3) sphenopetroclival; 4) foramen magnum; and 5) CPA. The authors pointed out the difficulty in grouping meningiomas into precise topographic areas because in some cases they are transitional.

There is lack of a uniform consensus on the definition of posterior petrous meningiomas; thus, it is difficult to compare data in the various published series. The lesions are frequently termed "CPA meningiomas" in the literature, which describes the tumor as occupying the CPA cistern. ${ }^{2,9-11,18-21}$ We believe, however, that this term is inaccurate, and does not precisely describe the point of dural attachment and predict the direction of cranial nerve displacement, which can significantly determine surgeryinduced morbidity.

Abbreviations used in this paper: AICA = anterior inferior cerebellar artery; $\mathrm{CPA}=$ cerebellopontine angle; IAC = internal auditory canal; MR = magnetic resonance; $\mathrm{VA}=$ vertebral artery; $\mathrm{VS}=$ vestibular schwannoma.
In their series Samii and Ammirati ${ }^{18}$ used the term "posterior pyramid meningiomas" and defined them as tumors whose main direction of growth brings them in contact with the posterior pyramid, irrespective of their site of dural attachment. They also designated those located anterior and those posterior to the internal acoustic meatus. Of the 56 lesions in their series, however, 18 arose from the tentorium, three from the clivus, and one from the jugular foramen. There were only nine cases in which the dural attachments originated from the petrous bone (six lesions anterior to and three lesions posterior to the internal acoustic meatus).

Al-Mefty ${ }^{1}$ described a group of CPA meningiomas as "petrosal meningiomas" that originated on the posterior surface of the petrous bone and divided them into two categories: tumors originating anterior to the internal auditory meatus and those originating posterior to it. Based on topography and displacement patterns of neurovascular structures, Bricolo and Turazzi ${ }^{3}$ classified meningiomas with dural attachments arising from the posterior surface of the petrous bone "posterior to the internal acoustic meatus as posterior petrous meningiomas." Lesions situated anterior to the internal acoustic meatus were named "anterior petrous meningiomas" (Fig. 1 left). In their experience, anterior petrous meningiomas tended to displace seventh and eighth cranial nerves posteriorly, whereas posterior petrous meningiomas displaced the nerves anteriorly. The anterior petrous meningiomas were more difficult to remove because the cranial nerves were situated between the surgeon and the tumor. 


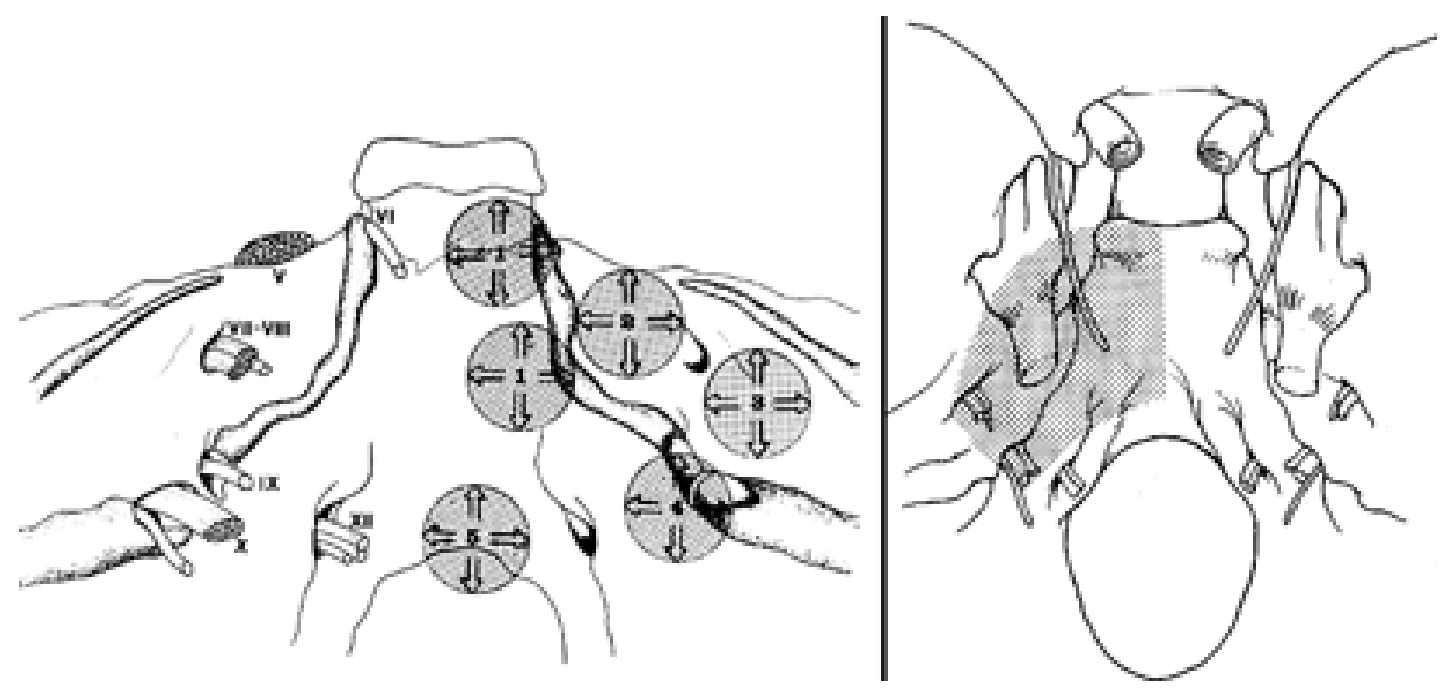

Fig. 1. Illustrations. Left: Illustration depicting the potential sites of dural attachment on the posterior skull base: 1) petroclival; 2) anterior petrous; 3) posterior petrous; 4) jugular foramen; and 5) foramen magnum. (From Bricolo and Turazzi; used with permission). Right: Petroclival meningiomas have dural attachments that originate at or medial to the skull base foramina of the fifth through 11th cranial nerves (shaded region). In contrast, posterior petrous meningiomas have dural attachments that arise lateral to these cranial nerve foramina. From Couldwell, et al., 1996; used with permission.

In this review, we define posterior petrous meningiomas as tumors with basal dural attachments originating on the petrous bone posterior to the internal auditory meatus and lateral to the skull base foramina of the fifth through 11th cranial nerves. These tumors may project to the CPA and mimic VSs, displacing neurovascular structures. The chance of totally removing posterior petrous meningiomas is excellent without significant morbidity as opposed to petroclival meningiomas. Petroclival meningiomas whose dural attachments emanate at or medial to the skull base foramina of the fifth through 11th cranial nerves (Fig. 1 right $)^{6}$ place the cranial nerves between the surgeon and the tumor, and are thus associated with significantly higher rates of surgical morbidity, primarily as a result of cranial neuropathies and vascular injury to the brainstem perforating vessels. ${ }^{6,17,21}$

\section{CLINICAL FEATURES}

The onset of clinical symptoms of posterior petrous meningiomas is insidious. Because these tumors often project into the CPA, patients may present with signs and symptoms of cranial neuropathy (fifth-11th cranial nerves), cerebellar dysfunction, and brainstem compression.

Of CPA tumors, VSs are the most common (75\%) followed by meningiomas (10\%). Most posterior petrous meningiomas are usually larger than the average VS at presentation. In contrast to VSs, auditory and vestibular dysfunction are not always the major presenting symptoms. Hearing loss, tinnitus, and disequilibrium, however, may be the initial symptoms in a patient with a meningioma arising near the porus acousticus. In the experience of Rhoton, ${ }^{16}$ the most common CPA meningioma originates above the internal auditory meatus, and this often presents with facial pain or other sensory symptoms before developing eighth cranial nerve symptoms. If the meningioma is attached below the internal auditory meatus and extends inferiorly near the jugular foramen, the patient may develop hoarseness and difficulty swallowing. In late stages of the disease course, patients may manifest diplopia, ataxia, papilledema, and hydrocephalus due to compression of the cerebellum and obstruction of the fourth ventricle.

Cushing and Eisenhardt ${ }^{8}$ noted that all their patients presented with hearing loss mimicking that related to VSs. Of their seven cases, five experienced facial weakness and four sensory disturbance due to trigeminal nerve involvement. Symptoms differ depending on the location of the tumor relative to the internal auditory meatus. ${ }^{20}$ In the review published by Samii and Ammirati, ${ }^{18}$ meningiomas that arose posterior to the internal auditory meatus usually manifested in patients at presentation as cerebellar dysfunction and gait ataxia, whereas those that arose anterior to the internal auditory meatus typically caused facial pain, facial numbness, and hearing loss.

The seventh-eighth cranial nerve complex is usually displaced anteriorly by posterior petrous meningiomas; less frequently, it may be pushed cranially or caudally. The fifth nerve is frequently displaced cranially or anteriorly. The lower cranial nerves are usually displaced caudally. ${ }^{18}$ Larger tumors may cause compression of the fourth ventricle, resulting in noncommunicating hydrocephalus. Some of these tumors originate low on the petrous pyramid at the jugular tubercle and may extend into the jugular foramen. ${ }^{1}$

\section{NEUROIMAGING CHARACTERISTICS}

Detailed neuroimaging studies are important for surgical planning and preoperatively defining the location of the tumor and its relationship to the cranial nerves and brainstem. Magnetic resonance imaging is the modality of choice. 
Most posterior petrous meningiomas are based broadly against the dural surface of the posterior medial petrous bone (Figs. 2 and 3). Many exhibit an obtuse angle as they interface with the posterior surface of the petrous bone. On MR imaging, they are well-circumscribed masses that are characteristically hypointense to isointense on $\mathrm{T}_{1}$ weighted sequences and isointense to hyperintense on $T_{2}$ weighted sequences. They enhance homogeneously and avidly after intravenous administration of Gd. A dural tail is frequently seen with meningiomas; however, some schwannomas and metastases may also have a dural tail caused by tumor infiltration and/or reactive changes. ${ }^{15}$

Computerized tomography scanning of the temporal bone may be useful for detailing osseous changes such as hyperostosis and erosion or expansion of the IAC. A widened IAC usually indicates a VS. ${ }^{21}$ Posterior petrous meningiomas rarely erode or extend into the IAC and occasionally may contain calcium or hyperostotic bone. Hyperostosis may not, however, be an obvious distinguishing feature because of the marked density of the petrous bone. Meningiomas are usually larger and oval shaped with a sessile broad base on the petrous bone. Vestibular schwannomas are smaller, round, and have a narrow attachment centered on the IAC. ${ }^{14,22}$

\section{SURGICAL ANATOMY}

The surgeon must possess a sound understanding of the anatomical features of the CPA and posterior skull base (Fig. 4). The CPA, by definition, is the space between the cerebellum and the pons. Its cistern is delimited by the pons medially and by the pyramid of the petrous bone laterally. ${ }^{18}$ It is limited superiorly by the tentorium, medially by the pons, and laterally by the posterior petrous ridge. There is no inferior limit because the CPA is continuous with the lateral cerebellomedullary cistern.

The relationship of the tumor to the cranial nerves provides an immediate understanding of the site of dural origin and, consequently, the related difficulties in its re- moval. Because posterior petrous meningiomas arise lateral to the cranial nerve foramina, they usually displace the fifth through eighth cranial nerves anteriorly. Petroclival meningiomas, on the other hand, arise medially to the cranial nerve foramina and thus tend to displace these nerves posteriorly.

Knowledge of the blood supply to these tumors is useful for surgical planning and tumor ablation. Posterior petrous meningiomas are usually fed by branches of the ascending pharyngeal artery, occipital artery, VA, subarcuate artery of the AICA, and middle meningeal artery. Pial branches of the anterior inferior cerebellar artery and superior cerebellar artery can be parasitized with larger tumors. ${ }^{13}$ In these types of tumors, preoperative embolization is generally not necessary.

\section{OPERATIVE APPROACHES}

\section{Retrosigmoid Approach}

The retrosigmoid (lateral suboccipital) approach is the most common selected route for removing lesions of the CPA. It is the preferred approach at our institution for removing posterior petrous meningiomas, especially if properative hearing is intact (Fig. 2). The retrosigmoid approach offers advantages of hearing preservation, early drainage of cerebrospinal fluid cisterns allowing for cerebellar relaxation, and early visualization of lower cranial nerves. $^{21}$

Intraoperative monitoring including somatosensory evoked potentials, brainstem auditory evoked potentials, and facial nerve monitoring should be implemented. On some occasions, the lower cranial nerves are monitored for resection of inferiorly extending tumors that involve the ninth through 11th cranial nerves.

Although various types of positioning can be used for this approach, such as the sitting position or supine position with the head rotated and the shoulder slightly elevated with a shoulder roll, we prefer to place the patient in the lateral position (Fig. 5 upper). The head is held in three-
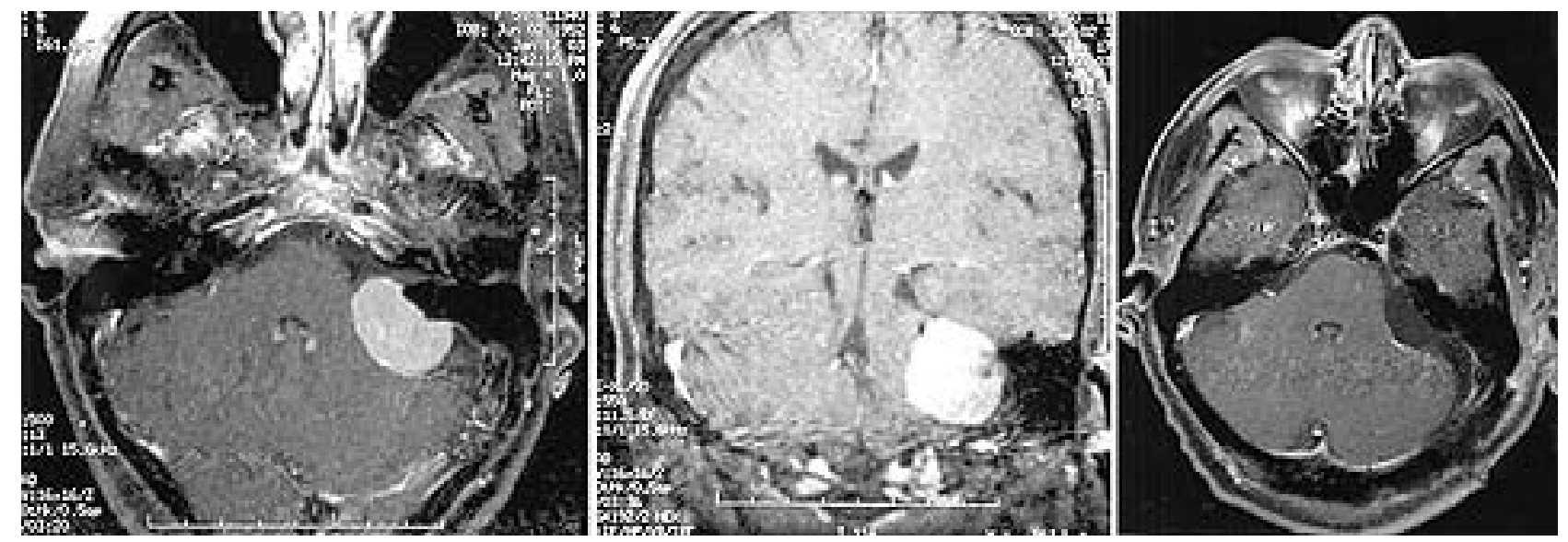

Fig. 2. Magnetic resonance images obtained in a 50-year-old woman who presented with a mild left-sided hearing loss, tinnitus, and ataxia. Left and Center: Axial (left) and coronal (center) Gd-enhanced images revealing an enhancing broad-based lesion against the posterior surface of the petrous bone. The seventh and eighth cranial nerves are displaced anteriorly. A dural tail is present at the posterolateral aspect of the tumor. Right: Postoperative axial image obtained after successful tumor excision via the retrosigmoid approach. 

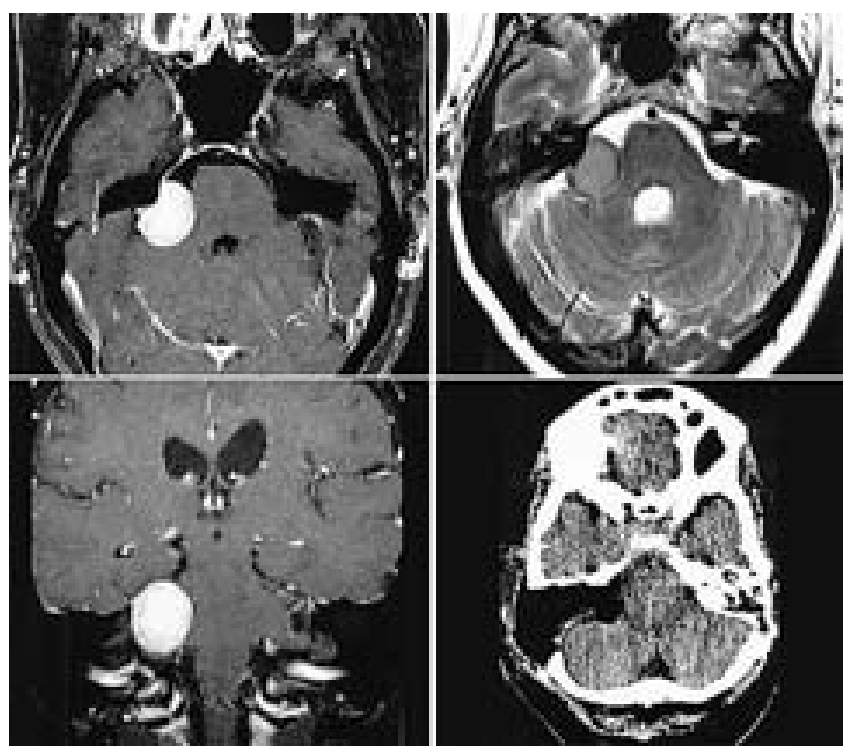

Fig. 3. Magnetic resonance images and computerized tomography scan obtained in a 55-year-old woman who presented with nonserviceable hearing loss and tinnitus in her right ear. Upper Left and Lower Left: Axial $\mathrm{T}_{1}$-weighted (upper left), axial $\mathrm{T}_{2}-$ weighted (upper right), and coronal $\mathrm{T}_{1}$-weighted (lower left) images revealing a posterior petrous meningioma. The enhancing lesion arises from the posterior surface of the petrous bone posterior to the IAC. The seventh and eighth cranial nerves are displaced anteriorly. Lower Right: Postoperative computerized tomography scan obtained after gross-total resection via the translabyrinthine approach.

point pin fixation and is oriented laterally with the vertex angled slightly down and the face slightly rotated ventral so that the external auditory meatus and the mastoid bone are at the highest point. An axillary roll is placed, and the contralateral arm rests on a Krauss armrest. The elevated arm is distracted inferiorly toward the foot of the table to provide more room for the surgeon above the shoulder. All pressure points are carefully padded with foam or gel pads. The patient is secured to the operating table with adhesive tape to allow safe rotation of the table during the operation, improving the surgeon's line of sight. The anesthetist is asked to administer glucocorticoid agents and mannitol at the time of the skin incision.

A retromastoid curvilinear skin incision is made approximately 1 to $2 \mathrm{~cm}$ behind the body of the mastoid (Fig. 5 lower). A fascial layer of pericranium is harvested to aid in later closure of the dura. The muscle opening is brought inferiorly to enable palpation of the foramen magnum and $\mathrm{C}-1$. Care is taken to avoid injury to the VA in its extradural course superior to the $\mathrm{C}-1$ arch. A craniectomy or craniotomy is made medial to the sigmoid sinus. The opening is enlarged to expose the region of the posterior petrous bone of interest. If the tumor is in a superior location close to the tentorium, the lower margin of the transverse sinus and the medial margin of the sigmoid sinus are exposed. Tumors located more inferiorly may not require exposure of the transverse sinus. Bone wax is applied to any exposed mastoid air cells in the lateral part of the craniectomy. The dura is usually opened and reflected medially. The dural cuff bordering the transverse and sigmoid

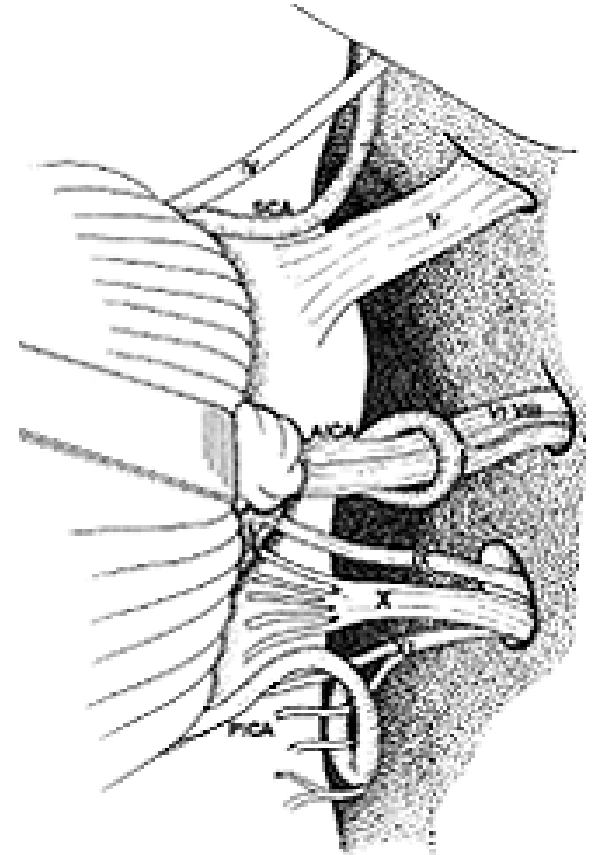

Fig. 4. Schematic illustration of the CPA. The cerebellum has been retracted to show (from top to bottom) the following: the trochlear nerve (IV), the superior cerebellar artery (SCA), the trigeminal nerve (V), facial and vestibulocochlear nerves (VII and VIII) with the AICA, the glossopharyngeal (IX), vagus (X), and spinal accessory (XI) nerves with the posterior inferior cerebellar artery (PICA). From Bricolo and Turazzi; used with permission.

sinuses is tacked up to optimize exposure. Alternatively, the dura may be opened with flaps that base on the transverse and sigmoid sinuses. Opening the arachnoid over the cisterna magna or the superolateral margin of the cerebellum allows cerebrospinal fluid to escape, providing cerebellar relaxation. The cerebellum is carefully retracted medially to expose the contents of the CPA.

The establishment of an arachnoid plane is critical for separating the tumor from the cerebellum and brainstem as well as microdissection of the neurovascular structures. ${ }^{1}$ Resection begins with progressive devascularization of the tumor by coagulating and dividing its vascular supply from the tentorium and from its attachment to the petrous pyramid. In all cases but those involving the smallest tumors, the tumor capsule should be opened and the lesion debulked centrally by using suction, microscopic sharp reduction, bipolar cautery, and/or ultrasonic aspiration. Intracapsular removal enables relaxation of the tumor capsule and should be completed before an attempt is made to separate the lesion from the compressed adjoining nerves and brainstem. The remaining thin layer of capsule is then gently separated from the brainstem and cranial nerves. Once the tumor is excised, its dural attachment is removed or coagulated and any hyperostotic bone is drilled away. Care should be taken not to violate the inner ear structures of the temporal bone.

In those cases in which the transverse or sigmoid sinus is involved with tumor, one must choose to sacrifice or repair the sinus. In these cases the senior author performs an MR venography study and, in some cases, catheter 


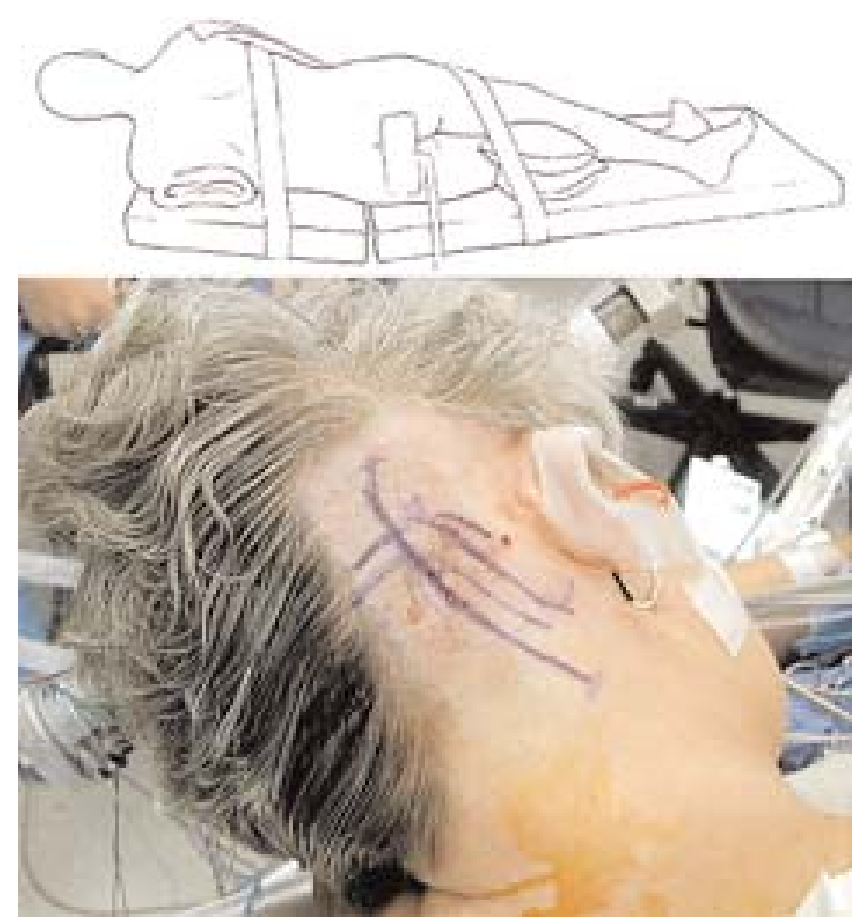

Fig. 5. Retrosigmoid approach. Upper: Illustration showing the lateral position. Lower: A retromastoid skin incision is made approximately $1 \mathrm{~cm}$ behind the body of the mastoid. The transverse and sigmoid sinuses are drawn to show their relationship to the skin incision.

angiography to evaluate the venous anatomy and determine if the transverse sinuses are both patent and to evaluate their communication at the torcula. If the sinus is involved with tumor and its preservation is desired, postresection repair will be undertaken primarily by using standard microvascular technique and suturing of a fascial or dural graft.

After tumor resection, the dura is closed watertight and a piece of previously harvested pericranium is used for grafting. The bone flap, if a craniotomy was performed, is fixed in place with a microplate, and the muscle is closed in multiple layers. Occasionally, porous polyethylene is used for a cranioplasty to cover the craniectomy defect and is fastened in place with titanium screws. ${ }^{7}$

\section{Transtemporal Approaches}

In selected cases where the patient possesses nonserviceable hearing, a translabyrinthine approach may occasionally be used (Fig. 3). This approach offers the advantages of early identification and control of the facial nerve before any dissection or removal of tumor, increased preservation of the anatomical and functional integrity of the facial nerve, and minimal cerebellar retraction. House, ${ }^{12}$ who popularized the translabyrinthine approach for VSs, has emphasized the vertical crest (Bill bar) at the lateral end of the IAC as a constant landmark. Giannotta, et al. ${ }^{10}$ have described two cases of CPA meningiomas that were successfully resected via the translabyrinthine approach.

The head is placed in the lateral position. A postauricular incision is made approximately 1 in behind the postau- ricular crease, extending from the mastoid tip to just above the pinna. The soft tissues are divided over the mastoid process, and the ear is reflected anteriorly. The root of the zygoma, spine of Henle, asterion, and mastoid tip serve as useful landmarks for the mastoidectomy.

Under microscopic visualization, the mastoid air cells are removed down to the mastoid antrum by using a highspeed cutting burr. The mastoid antrum serves as an important landmark because it defines the anterior limit of bone removal and locates the lateral semicircular canal. The sigmoid sinus is then skeletonized down to the jugular bulb. The posterior fossa dura medial to the sigmoid sinus, superior to the jugular bulb, and inferior to the superior petrosal sinus (Trautmann triangle) is exposed. Using a diamond burr, the semicircular canals are identified and drilled away to their entrance in the vestibule.

After opening the vestibule, the facial nerve, which lies anterior to these structures, is carefully skeletonized. Bone around the IAC is then removed. The transverse crest separating the superior and inferior division of the vestibular nerves can be seen. Identification of the facial nerve in the lateral end of the IAC is then accomplished by palpation of the Bill bar. The dura is opened in a Y-shaped fashion, starting from the sinodural angle near the sigmoid sinus and continuing down to the porus acousticus. A second incision is made from porus acousticus down toward the jugular bulb. Tumor removal proceeds as described in the previous section.

If a more lateral approach is desired in cases in which hearing is intact, a retrolabyrinthine transtemporal approach, rather than the standard retrosigmoid approach may be performed to devascularize the tumor and reduce the amount of cerebellar retraction necessary.

\section{Surgery-Related Results}

In a recent 10-year experience, 21 patients who harbored posterior petrous meningiomas (in accordance with the aforementioned strict anatomical definition) were surgically treated. Seventeen patients were women and four were men, whose mean age at presentation was 59 years (range 31-74 years). The most common presenting symptom was hearing loss (43\%). Tinnitus, imbalance, and facial numbness were the next common symptoms (Table 1).

The majority of these tumors were surgically removed via the retrosigmoid approach (Table 2). In two cases in which hearing was nonserviceable, the translabyrinthine approach was used, and in two cases in which there was extensive basal involvement and tumor extending above the tentorium, the petrosal approach ${ }^{5}$ was used. All procedures were performed with somatosensory evoked potential, brainstem auditory evoked potential, and facial nerve monitoring. Gross-total resection was achieved in 18 patients $(86 \%)$. In three patients, a subtotal resection was performed because the tumor adhered to the cranial nerves: in two patients it adhered to the ninth and 10th cranial nerves at the jugular foramen and in one to the seventh and eighth cranial nerves in the IAC. Minimal residual tumor was left behind.

The surgery-related complications are listed in Table 3. There were no deaths. The major postoperative deficit was transient facial nerve palsy (five cases). Three of these patients had regained full function after 1 year. None de- 
J. K. Liu, O. N. Gottfried, W. T. Couldwell

TABLE 1

Clinical presentation of 21 patients with posterior petrous meningiomas

\begin{tabular}{lc}
\hline \hline Signs or Symptoms & No. of Cases \\
\hline general & \\
headache, nausea, vomiting & 7 \\
trigeminal nerve & 2 \\
$\quad$ pain & 4 \\
$\quad$ numbness & 0 \\
diplopia & \\
facial nerve & 0 \\
$\quad$ paresis & 1 \\
spasm & \\
vestibulocochlear nerve & 6 \\
$\quad$ tinnitus & 9 \\
hearing loss & 6 \\
imbalance & 1 \\
dysphagia & 3 \\
ataxia & \\
hemiparesis & 0 \\
\hline
\end{tabular}

veloped new sensorineural hearing loss as a result of surgery (excluding translabyrinthine removal). Three patients developed transient vocal cord paresis, two of whom required vocal cord medialization.

\section{CONCLUSIONS}

The surgeon must possess a sound understanding of the anatomical features of the posterior petrous skull base and CPA. The relationship of the tumor to the cranial nerves provides an immediate understanding of the site of dural origin and, consequently, the related difficulties in its removal. Because posterior petrous meningiomas arise lateral to the cranial nerve foramina and, therefore, usually displace the fifth through 11th cranial nerves anteriorly. Because petroclival meningiomas, on the other hand, arise medial to the cranial nerve foramina, they usually tend to displace these nerves posteriorly. Using this strict definition for posterior petrous meningiomas, tumors presenting in this location are much less likely to result in surgical morbidity or mortality after removal. The position of attachment lateral to the cranial nerve foramina in the posterior petrous region renders more proximal tumor skull base attachment and easier surgical access. In addition, the tumor is proximal to the nerves when approached via a lateral approach, thus reducing cranial nerve manipulation and morbidity associated with tumor excision. The tumor is less likely to have intimate attachment to paramedian brainstem perforators. In the vast majority of cases, the

TABLE 2

Surgical approaches used in 21 patients with posterior petrous meningiomas

\begin{tabular}{lc}
\hline \hline \multicolumn{1}{c}{ Approach } & No. of Tumors \\
\hline retrosigmoid & 17 \\
translabyrinthine & 2 \\
petrosal & 2 \\
\hline
\end{tabular}

TABLE 3

Surgery-related complications in 21 patients with posterior petrous meningiomas*

\begin{tabular}{cc}
\hline \hline Complication & No. of Complications \\
\hline hemiparesis & 0 \\
postop hematoma & 0 \\
CN neuropathies & 0 \\
III & 0 \\
IV & 0 \\
V & 1 \\
VI & 5 \\
VII & 0 \\
VIII & 3 \\
vocal cord paresis &
\end{tabular}

$* \mathrm{CN}=$ cranial nerve.

tumor may be resected completely and safely via a simple retrosigmoid approach.

\section{References}

1. Al-Mefty O: Operative Atlas of Meningiomas. Philadelphia: Lippincott-Raven, 1998

2. Batra PS, Dutra JC, Wiet RJ: Auditory and facial nerve function following surgery for cerebellopontine angle meningiomas. Arch Otolaryngol Head Neck Surg 128:369-374, 2002

3. Bricolo A, Turazzi S: Petroclival meningiomas, in Schmidek $\mathrm{HH}$ (ed): Schmidek and Sweet Operative Neurosurgical Techniques: Indications, Methods, and Results, ed 4. Philadelphia: WB Saunders, 2000, pp 933-955

4. Castellano F, Ruggiero G: Meningiomas of the posterior fossa. Acta Radiol (Suppl) 104:1-164, 1953

5. Couldwell WT: Petrosal approach for resection of petroclival meningiomas, in Rengachary SS, Wilkins RH (eds): Neurosurgical Operative Atlas. Park Ridge, IL: AANS Publications, 1998, Vol 7, pp 69-81

6. Couldwell WT, Fukushima T, Giannotta SL, et al: Petroclival meningimoas: surgical experience in 109 cases. J Neurosurg 84:20-28, 1996

7. Couldwell WT, Stillerman CB, Dougherty W: Reconstruction of the skull base and cranium adjacent to sinuses with porous polyethylene implant: preliminary report. Skull Base Surg 7: 57-63, 1997

8. Cushing H, Eisenhardt L: Meningiomas. Their Classification, Regional Behavior, Life History, and Surgical End Results. Springfield, IL: Charles C Thomas, 1938, pp 3-387

9. Daspit CP, Spetzler RF, Pappas CT: Combined approach for lesions involving the cerebellopontine angle and skull base: experience with 20 cases - preliminary report. Otolaryngol Head Neck Surg 105:788-796, 1991

10. Giannotta SL, Pulec JL, Goodkin R: Translabyrinthine removal of cerebellopontine angle meningiomas. Neurosurgery 17: 620-625, 1985

11. Granick MS, Martuza RL, Parker SW, et al: Cerebellopontine angle meningiomas: clinical manifestations and diagnosis. Ann Otol Rhinol Laryngol 94:34-38, 1985

12. House WF: Transtemporal bone microsurgical removal of acoustic neuromas. Arch Otolaryngol 80:597-756, 1964

13. McDermott MW, Wilson CB: Meningiomas, in Youmans JR (ed): Neurological Surgery, ed 4. Philadelphia: WB Saunders, 1996, pp 2782-2825

14. Moller A, Hatam A, Olivecrona $\mathrm{H}$ : The differential diagnosis of pontine angle meningioma and acoustic neuroma with computed tomography. Neuroradiology 17:21-23, 1978 


\section{Posterior petrous meningiomas}

15. Osborn AG: Diagnostic Neuroradiology. St. Louis: Mosby, 1994

16. Rhoton AL Jr: Meningiomas of the cerebellopontine angle and foramen magnum. Neurosurg Clin N Am 5:349-377, 1994

17. Roberti F, Sekhar LN, Kalavakonda C, et al: Posterior fossa meningiomas: surgical experience in 161 cases. Surg Neurol 56:8-21, 2001

18. Samii M, Ammirati M: Cerebellopontine angle meningiomas (posterior pyramid meningiomas), in Al-Mefty O (ed): Meningiomas. New York: Raven Press, 1991, pp 503-511

19. Schaller B, Heilbronner R, Pfaltz CR, et al: Preoperative and postoperative auditory and facial nerve function in cerebellopontine angle meningiomas. Otolaryngol Head Neck Surg 112:228-234, 1995

20. Schaller B, Merlo A, Gratzl O, et al: Premeatal and retromeatal cerebellopontine angle meningioma. Two distinct clinical entities. Acta Neurochir 141:465-471, 1999
21. Sekhar LN, Jannetta PJ: Cerebellopontine angle meningiomas. Microsurgical excision and follow-up results. J Neurosurg 60: 500-505, 1984

22. Valavanis A, Schubiger O, Hayek J, et al: CT of meningiomas on the posterior surface of the petrous bone. Neuroradiology 22:111-121, 1981

23. Yaşargil MG, Mortara RW, Curcic M: Meningiomas of basal posterior cranial fossa. Adv Tech Stand Neurosurg 7:3-115, 1980

Manuscript received April 21, 2003.

Accepted in final form May 22, 2003.

Address reprint requests to: William T. Couldwell, M.D., Ph.D., Department of Neurosurgery, University of Utah, 30 North 1900 East, Suite 3B409, Salt Lake City, Utah 84132. email: william.couldwell@hsc.utah.edu. 